\title{
EFEITO DA INTERAÇÃO DO NICOSULFURON E CHLORPYRIFOS SOBRE O BANCO DE SEMENTES E OS ATRIBUTOS MICROBIANOS DO SOLO ${ }^{(1)}$
}

\author{
Taciane Almeida de Oliveira ${ }^{(2)}$, José Barbosa dos Santos ${ }^{(3)}$, Gessimar Nunes \\ Camelo $^{(4)}$, Rafael Grossi Botelho ${ }^{(5)}$ \& Thiago Magalhães de Lázari ${ }^{(6)}$
}

\begin{abstract}
RESUMO
Considerando o período de competição de plantas daninhas e a incidência da lagarta-do-cartucho na cultura do milho, há necessidade de aplicação, em curto intervalo de tempo, de herbicidas e de inseticidas, principalmente o nicosulfuron e o chlorpyrifos. $O$ objetivo deste estudo foi avaliar o efeito da aplicação sequencial do nicosulfuron e do chlorpyrifos sobre a emergência de plântulas do banco de sementes, a taxa de desprendimento de $\mathrm{CO}_{2}$ (respiração basal) e o $\mathrm{C}$ da biomassa microbiana (CBM) do solo. Foi realizada aplicação sequencial, em solo, do nicosulfuron (doses de 0 a $64 \mathrm{~g} \mathrm{ha}^{-1}$ ) associado ou não ao chlorpyrifos (0 e $\left.240 \mathrm{~g} \mathrm{ha}^{-1}\right)$. Aos 20, 40 e 60 dias após a aplicação (DAA) dos produtos, todas as plântulas emergidas do banco de sementes foram identificadas em nível de espécie, sendo estimadas a frequência, densidade e abundância, além do índice de valor de importância (IVI). Aos 60 DAA, determinou-se também a taxa de desprendimento de $\mathrm{CO}_{2}$, o $\mathrm{CBM}$ e o quociente metabólico $\left(\mathrm{qCO}_{2}\right)$, por meio da relação entre o $\mathrm{CO}_{2}$ acumulado e o CBM total do solo. A aplicação alterou severamente a massa de plântulas secas e o número de espécies nas doses superiores a $20 \mathrm{~g} \mathrm{ha}^{-1}$ do nicosulfuron. Na presença do herbicida, as espécies com maior IVI foram Boehavia diffusa e Commelina benghalensis. Quanto aos bioindicadores do solo, foi observado decréscimo na taxa da respiração basal do solo com o aumento da dose aplicada do nicosulfuron associado ao chlorpyrifos, sem efeito na ausência do inseticida. Houve decréscimo linear no CBM em todos os casos, independentemente da aplicação do chlorpyrifos; entretanto, observou-se uma taxa de decréscimo 4,5 vezes maior para o solo que recebeu esse inseticida em conjunto com o nicosulfuron. A avaliação do $\mathrm{qCO}_{2}$ confirmou o efeito negativo da aplicação do inseticida e do herbicida.
\end{abstract}

\footnotetext{
(1) Recebido para publicação em setembro de 2008 e aprovado em março de 2009.

(2) Mestranda em Entomologia, Laboratório de Pedobiologia, Instituto Nacional de Pesquisas da Amazônia - INPA. Av. Efigênio Sales 2239, Aleixo, CEP 69060-020 Manaus (AM). E-mail: taciane.almeida@yahoo.com.br

(3) Professor do Departamento de Agronomia da Universidade Federal dos Vales do Jequitinhonha e Mucuri - UFVJM. Campus II, Rod. MGT 367, Km 583, CEP 39100-000 Diamantina (MG). E-mail: jbarbosa@ufvjm.edu.br

(4) Mestrando em Fitotecnia, Departamento de Fitotecnica, Universidade Federal de Viçosa - UFV. Av. PH Rolfs s/n. CEP 36570000 Viçosa (MG). E-mail: gessimarcamelo@yahoo.com.br

(5) Pós-Graduando do curso de Ciências Biológicas, Universidade Vale do Rio Doce - UNIVALE. Rua Israel Pinheiro 2000, CEP 35020-520 Governador Valadares (MG). E-mail: rgrossibio@bol.com.br

(6) Engenheiro-Agrônomo, UNIVALE. E-mail: thiagolazari@yahoo.com.br
} 
Conclui-se que a aplicação de chlorpyrifos + nicosulfuron promove impacto negativo sobre o banco de sementes e sobre a atividade microbiana do solo.

Termos de indexação: agrotóxico, carbono da biomassa microbiana, impacto ambiental, sinergismo.

\title{
SUMMARY: EFFECT OF SEQUENTIAL NICOSULFURON AND CHLORPYRIFOS APPLICATION ON SEED BANK AND SOIL MICROBIAL CHARACTERISTICS
}

\begin{abstract}
In the period of competition of weeds and the incidence of fall armyworm in the corn crop there is a need for herbicide and insecticide such as nicosulfuron and chlorpyrifos application within short time intervals. The aim of this study was to evaluate the effect of sequential applications of nicosulfuron and chlorpyrifos on the emergence of seedlings of the seed bank in the soil, the basal $\mathrm{CO}_{2}$ emission rate, and the microbial biomass carbon (MBC) of soil.

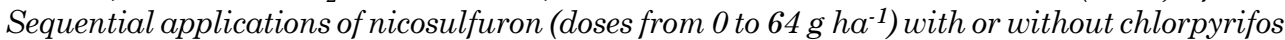
(0 and $240 \mathrm{~g} \mathrm{ha}^{-1}$ ) were performed. At 20, 40 and 60 days after application (DAA) of the products, the species of all seedlings that emerged from the seed bank were identified, and the frequency, density and abundance estimated, as well as the importance value IV. Sixty DAA the $\mathrm{CO}_{2}$ emission rate and $C B M$ were were also determined, and based on the relationship between the accumulated $\mathrm{CO}_{2}$ and total soil $\mathrm{MBC}$ the metabolic coefficient $\left(q \mathrm{CO}_{2}\right)$ was estimated. The application of nicosulfuron rates of over $20 \mathrm{~g} \mathrm{ha}^{-1}$ severely affected the seedling dry weight and number of species. In the presence of the herbicide, the species with highest IV were Boehavia diffusa and Commelina bengalensis. There was a decrease in the basal soil respiration rate with increasing nicosulfuron doses, in the presence as well as in the absence of the insecticide chlorpyrifos. There was a linear decrease in MBC in all cases regardless of the chlorpyrifos application, although the reduction was 4.5 times greater in soil that received the combined application of the insecticide and nicosulfuron. The $q \mathrm{CO}_{2}$ confirmed the negative effect of the application of insecticide and herbicide. It was concluded that the application of chlorpyrifos + nicosulfuron causes a negative impact on the seeds in the soil and the soil microbial activity.
\end{abstract}

Index terms: pesticides, microbial biomass carbon, environmental impact, synergism.

\section{INTRODUÇÃO}

Plantas, animais e microrganismos que vivem em determinada área estão estreitamente ligados por uma rede complexa de relações, afetadas direta ou indiretamente pelas atividades agrícolas, nos seus mais diferentes segmentos (Moreira et al., 1996). Nesse sentido, a aplicação de agrotóxicos no compartimento solo tem papel marcante na mudança de ecossistemas. Considerando o período crítico de competição de plantas daninhas e a incidência da lagarta-do-cartucho na cultura do milho, é observada a necessidade de aplicação, em curto intervalo de tempo, de herbicidas e de inseticidas, com destaque para o nicosulfuron e o chlorpyrifos, respectivamente (Silva et al., 2005). Com a introdução do sistema de integração lavoura-pecuária e demais sistemas de consorciação, esses produtos vêm sendo utilizados de maneira integrada, em razão da necessidade de diminuição dos custos de aplicação.

Nesse contexto, pesquisas já apresentaram resultados negativos da aplicação da mistura entre o herbicida nicosulfuron e o inseticida chlorpyrifos, quando aplicados simultaneamente sobre plantas de milho em consórcio com braquiária (Pereira et al., 2005; Silva et al., 2005; Nicolai et al., 2006). Pelo efeito sinergístico da mistura, o herbicida, antes seletivo ao milho, apresenta efeitos tóxicos sobre este. No entanto, não foram avaliadas as consequências dessa prática no que se refere às espécies presentes na área sob a forma de sementes no solo (banco de sementes do solo). $\mathrm{O}$ banco de sementes fornece informações sobre as condições ambientais e práticas culturais anteriores, sendo importante fator na determinação do suprimento de novos indivíduos para as comunidades locais (Templeton \& Levin, 1979a; Monquero \& Christoffoletti, 2005).

Uma proposta para predizer os efeitos negativos de práticas agrícolas no solo e na água é o uso de bioindicadores, preferencialmente os microbianos (Tótola \& Chaer, 2002). Nesse sentido, a atividade microbiana do solo pode fornecer informações sobre a estabilidade da comunidade de organismos, visto a sua comprovada sensibilidade a distúrbios no solo provocados por diferentes sistemas de manejo (Chaer \& Tótola, 2007; Pereira et al., 2007; Rangel \& Silva, 2007).

A avaliação da taxa de respiração, e a quantificação da biomassa microbiana e da relação entre estes, denominada quociente metabólico $\left(\mathrm{qCO}_{2}\right)$, estão diretamente relacionadas ao fato de que, à medida que os microrganismos do solo se tornam mais eficientes na utilização dos recursos do ecossistema, menor 
quantidade de $\mathrm{C}$ se perde como $\mathrm{CO}_{2}$ pela respiração e maior proporção é incorporada às células microbianas (Anderson \& Domsch, 1985). O efeito tóxico dos produtos nicosulfuron e chlorpyrifos nos microrganismos do solo já foi relatado em outros trabalhos (Oliveira et al., 2003; Jakelaitis et al., 2007).

Dessa forma, o objetivo deste trabalho foi avaliar o efeito da aplicação sequencial do nicosulfuron e do chlorpyrifos sobre a emergência de plântulas do banco de sementes e sobre os microrganismos do solo.

\section{MATERIAL E MÉTODOS}

O trabalho foi realizado em casa de vegetação e laboratório pertencente à Faculdade de Ciências Agrárias-FAAG da Universidade Vale do Rio Doce UNIVALE. Amostras de solo foram coletadas em área com histórico de cultivo do milho, porém sem resíduo de herbicidas e, ou, inseticidas nos últimos três anos. Por ocasião dessa coleta, a vegetação predominante foi constituída por Boehavia diffusa, Commelina benghalensis e Euphorbia heterophylla, todas em estádio inicial de desenvolvimento. Após a coleta das amostras, na profundidade de $0-10 \mathrm{~cm}$ e peneiramento em malha de $4 \mathrm{~mm}^{2}$, foram preenchidos vasos de PVC com capacidade para $2 \mathrm{~L}$, previamente forrados com sacos plásticos. Visando à expressão dos resultados em número de plântulas por $\mathrm{m}^{2}$, estabeleceu-se, para cada unidade experimental (vaso), uma profundidade de $10 \mathrm{~cm}$ de substrato, correspondendo àquela camada retirada no campo. Os tratamentos foram compostos pela combinação de seis doses do herbicida nicosulfuron $\left(0,8,16,32,48\right.$ e $\left.64 \mathrm{~g} \mathrm{ha}^{-1}\right)$ e duas doses do inseticida (0 e $240 \mathrm{~g} \mathrm{ha}^{-1}$ ), aplicadas separadamente. O delineamento adotado foi inteiramente casualizado, com quatro repetições, totalizando 48 vasos.

Para aplicação dos agrotóxicos, foi utilizado um pulverizador costal pressurizado com $\mathrm{CO}_{2}$, aplicando o equivalente a $200 \mathrm{~L} \mathrm{ha}^{-1}$ de calda. Aos 20,40 e 60 dias da aplicação, as plântulas emergidas foram coletadas e classificadas quanto a família, gênero e espécie, sendo, posteriormente, secas em estufa $\left(75^{\circ} \mathrm{C} / 72 \mathrm{~h}\right) \mathrm{e}$ pesadas, com valores de massa total expressos em gramas por unidade de área. Após cada avaliação, aos 20 e 40 dias, os substratos nos vasos eram revolvidos, possibilitando, assim, a emergência de novas plântulas.

Após a classificação final das plantas, a partir dos dados de espécies totais presentes por tratamento, foram estimados frequência (distribuição das espécies nas unidades experimentais), densidade (quantidade de plantas de cada espécie por unidade de área, abundância (concentração das espécies em função dos tratamentos), frequência relativa, densidade relativa e abundância relativa (relação de cada espécie com as outras espécies encontradas na área) e índice de valor de importância (importância das espécies dentro da área estudada) (Mueller-Dombois \& Ellenberg, 1974).
Aos 60 DAA, foram retiradas amostras de solo em cada unidade experimental, para determinação dos indicadores microbianos. A atividade microbiana foi estimada a partir da quantidade de $\mathrm{CO}_{2}$ liberado por amostras de $150 \mathrm{~g}$ do solo de cada vaso, o qual foi capturado em frascos contendo $20 \mathrm{~mL}$ de $\mathrm{NaOH}(0,5$ mol L ${ }^{-1}$ ), em sistema fechado. As amostras de solo, após secas ao ar e peneiradas em malha de $4 \mathrm{~mm}^{2}$, foram submetidas à determinação do teor de água $\mathrm{e}$ incubadas por 17 dias com umidade em torno de $65 \%$ da capacidade de campo. Após incubação, procedeu-se à titulação do $\mathrm{NaOH}$ com $\mathrm{HCl}\left(0,25 \mathrm{~mol} \mathrm{~L}^{-1}\right)$, determinando-se o excesso de $\mathrm{NaOH}$ que não reagiu com o $\mathrm{CO}_{2}$ liberado (Anderson, 1982).

No final do período de incubação das amostras de solos, foram determinados o $\mathrm{C}$ da biomassa microbiana (CBM), pelo método descrito por Vance et al. (1987), utilizando, em lugar do clorofórmio (fumigação), forno de micro-ondas (irradiação) (Islam \& Weil, 1998; Ferreira et al., 1999), e o quociente metabólico $\left(\mathrm{qCO}_{2}\right)$, por meio da relação entre o $\mathrm{CO}_{2}$ acumulado e o $\mathrm{C}$ total da biomassa microbiana.

Com as médias obtidas dos tratamentos, fez-se análise de variância, sendo determinadas regressões relacionando doses do herbicida na presença e ausência de inseticida, produção de massa seca de plântulas e indicadores microbiológicos do solo. As significâncias das equações de regressão foram testadas pelo teste $t$ (1\%).

\section{RESULTADOS}

Considerando as três épocas de amostragem das plântulas (20, 40 e 60 dias), em relação ao aumento da dose de herbicida aplicada, foi observado decréscimo exponencial na massa total seca, sendo maior que $50 \%$ a partir da dose aproximada de $8 \mathrm{~g} \mathrm{ha}^{-1}$ (Figura 1). A

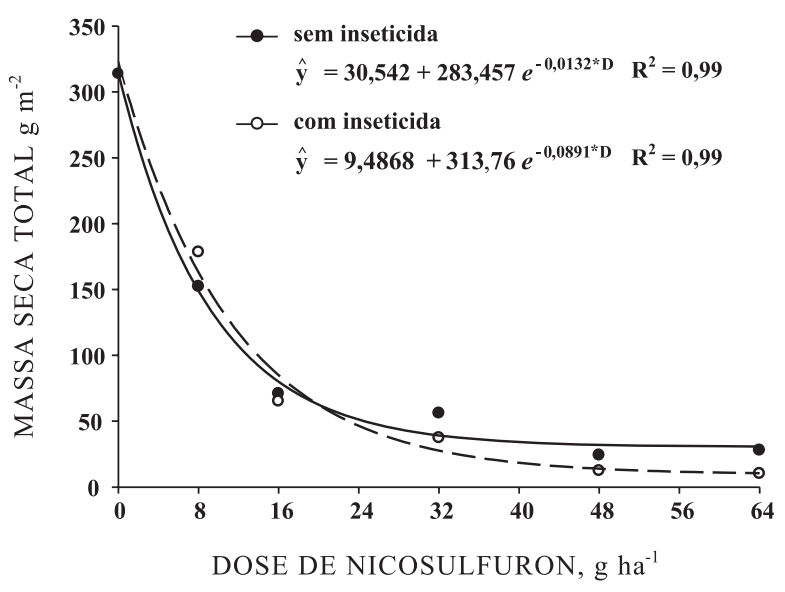

Figura 1. Massa total de plântulas secas provenientes do banco de sementes, coletadas aos 20,40 e 60 dias após a aplicação de doses do nicosulfuron com e sem a aplicação simultânea do inseticida chlorpyrifos. 
mistura ao chlorpyrifos não provocou grande mudança nesse comportamento até a dose aproximada de $20 \mathrm{~g} \mathrm{ha}^{-1}$, a partir da qual a massa seca foi reduzida acentuadamente (Figura 1).
Quanto às características fitossociológicas (Quadro 1), pode-se observar o efeito residual do herbicida, considerando a acentuada diminuição do número total de indivíduos (NTI) das parcelas que

Quadro 1. Principais espécies de plantas daninhas e respectivas características fitossociológicas, provenientes do banco de sementes, emergidas em solo submetido a diferentes doses do nicosulfuron com e sem a aplicação simultânea do inseticida chlorpyrifos. Avaliação resultante do somatório de três épocas de amostragem (20, 40 e 60 dias após aplicação dos produtos)

\begin{tabular}{|c|c|c|c|c|c|c|c|c|c|}
\hline Plântula ${ }^{(1)}$ & NTI & NPP & FRE & DEN & $\mathrm{ABU}$ & FRR & DER & $\mathrm{ABR}$ & IVI \\
\hline 20 espécies & \multicolumn{9}{|c|}{ Testemunha: $0,0 \mathrm{~g} \mathrm{ha}^{-1}$ de chlorpyrifos $+0,0 \mathrm{~g} \mathrm{ha}^{-1}$ de nicosulfuron } \\
\hline Boehavia diffusa & 92 & 4 & 1,00 & $3285,71^{\circ}$ & 23,00 & 6,45 & 17,76 & 16,74 & 40,95 \\
\hline Mollugo verticillata & 133 & 4 & 1,00 & 4750,00 & 33,25 & 6,45 & 25,68 & 24,20 & 56,32 \\
\hline Outras espécies & 293 & 54 & 13,5 & 10464,27 & 81,17 & 87,09 & 56,57 & 59,07 & 202,72 \\
\hline 13 espécies & \multicolumn{9}{|c|}{$0,0 \mathrm{~g} \mathrm{ha}^{-1}$ de chlorpyrifos $+8,0 \mathrm{~g} \mathrm{ha}^{-1}$ de nicosulfuron } \\
\hline Boehavia diffusa & 99 & 4 & 1,00 & 3535,71 & 24,75 & 12,50 & 55,62 & 44,26 & 112,38 \\
\hline Commelina benghalensis & 18 & 4 & 1,00 & 642,86 & 4,50 & 12,50 & 10,11 & 8,05 & 30,66 \\
\hline Outras espécies & 61 & 24 & 6 & 2178,57 & 26,67 & 75,03 & 34,28 & 47,7 & 156,96 \\
\hline 14 espécies & \multicolumn{9}{|c|}{$0,0 \mathrm{~g} \mathrm{ha}^{-1}$ de chlorpyrifos $+16,0 \mathrm{~g} \mathrm{ha}^{-1}$ de nicosulfuron } \\
\hline Boehavia diffusa & 77 & & 41,00 & 2750,00 & 19,25 & 13,79 & 61,60 & 45,21 & 120,60 \\
\hline Commelina benghalensis & 12 & 3 & 0,75 & 428,57 & 4,00 & 10,34 & 9,60 & 9,39 & 29,34 \\
\hline Outras espécies & 36 & 22 & 5,5 & 1285,69 & 19,33 & 75,88 & 28,8 & 45,4 & 150,08 \\
\hline 15 espécies & \multicolumn{9}{|c|}{$0,0 \mathrm{~g} \mathrm{ha}^{-1}$ de chlorpyrifos $+32,0 \mathrm{~g} \mathrm{ha}^{-1}$ de nicosulfuron } \\
\hline Boehavia diffusa & 76 & 4 & $1,00^{\delta}$ & 2714,29 & 19,00 & 14,29 & 59,37 & 42,38 & 116,04 \\
\hline Commelina benghalensis & 16 & 3 & 0,75 & 571,43 & 5,33 & 10,71 & 12,50 & 11,90 & 35,11 \\
\hline Outras espécies & 36 & 21 & 5,25 & 1285,69 & 20,5 & 74,97 & 28,1 & 45,72 & 148,83 \\
\hline 11 espécies & \multicolumn{9}{|c|}{$0,0 \mathrm{~g} \mathrm{ha}^{-1}$ de chlorpyrifos $+48,0 \mathrm{~g} \mathrm{ha}^{-1}$ de nicosulfuron } \\
\hline Euphorbia heterophylla & 13 & 3 & 0,75 & 464,29 & 4,33 & 13,04 & 14,94 & 13,83 & 41,82 \\
\hline Boehavia diffusa & 42 & 4 & 1,00 & 1500,00 & 10,50 & 17,39 & 48,28 & 33,51 & 99,18 \\
\hline Outras espécies & 32 & 16 & 4 & 1142,85 & 16,5 & 69,58 & 36,79 & 52,65 & 159,01 \\
\hline $\begin{array}{c}8 \text { espécies } \\
\text { Guphorbioteranhvlla }\end{array}$ & \multicolumn{9}{|c|}{$0,0 \mathrm{~g} \mathrm{ha}^{-1}$ de chlorpyrifos $+64,0 \mathrm{~g} \mathrm{ha}^{-1}$ de nicosulfuron } \\
\hline Boehavia diffusa & 62 & 4 & 1,00 & 2214,29 & 15,50 & 20,00 & 65,26 & 50,96 & 136,22 \\
\hline Outras espécies & 24 & 12 & 3 & 857,14 & 12,67 & 60 & 25,26 & 41,65 & 126,9 \\
\hline 17 espécies & \multicolumn{9}{|c|}{$240,0 \mathrm{~g} \mathrm{ha}^{-1}$ de chlorpyrifos $+0,0 \mathrm{~g} \mathrm{ha}^{-1}$ de $\mathrm{n}$ icosulfuron } \\
\hline Amaranthus hybridus & 56 & 4 & 1,00 & 2000,00 & 14,00 & 7,84 & 14,11 & 12,38 & 34,33 \\
\hline Mollugo verticillata & 92 & 4 & 1,00 & 3285,71 & 23,00 & 7,84 & 23,17 & 20,34 & 51,36 \\
\hline Outras espécies & 249 & 43 & 10,75 & 8892,83 & 76,09 & 84,28 & 62,72 & 67,27 & 214,34 \\
\hline 15 espécies & \multicolumn{9}{|c|}{$240,0 \mathrm{~g} \mathrm{ha}^{-1}$ de chlorpyrifos $+8,0 \mathrm{~g} \mathrm{ha}^{-1}$ de nicosulfuron } \\
\hline Boehavia diffusa & 76 & 4 & 1,00 & 2714,29 & 19,00 & 13,33 & 54,68 & 36,19 & 104,20 \\
\hline Commelina benghalensis & 13 & 3 & 0,75 & 464,29 & 4,33 & 10,00 & 9,35 & 8,25 & 27,61 \\
\hline Outras espécies & 50 & 23 & 5,75 & 1785,71 & 29,17 & 76,67 & 36 & 55,54 & 168,19 \\
\hline 18 espécies & \multicolumn{9}{|c|}{$240,0 \mathrm{~g} \mathrm{ha}^{-1}$ de chlorpyrifos $+16,0 \mathrm{~g} \mathrm{ha}^{-1}$ de nicosulfuron } \\
\hline Boehavia diffusa & 60 & 4 & 1,00 & 2142,86 & 15,00 & 10,26 & 45,45 & 31,80 & 87,51 \\
\hline Commelina benghalensis & 19 & 4 & 1,00 & 678,57 & 4,75 & 10,26 & 14,39 & 10,07 & 34,72 \\
\hline Outras espécies & 53 & 31 & 7,75 & 1892,83 & 27,42 & 79,46 & 40,16 & 58,12 & 177,76 \\
\hline 13 espécies & \multicolumn{9}{|c|}{$240,0 \mathrm{~g} \mathrm{ha}^{-1}$ de chlorpyrifos $+32,0 \mathrm{~g} \mathrm{ha}^{-1}$ de nicosulfuron } \\
\hline Boehavia diffusa & 65 & 4 & 1,00 & 2321,43 & 16,25 & 14,29 & 55,56 & 41,58 & 111,42 \\
\hline Commelina benghalensis & 16 & 3 & 0,75 & 571,43 & 5,33 & 10,71 & 13,68 & 13,65 & 38,04 \\
\hline Outras espécies & 36 & 21 & 5,25 & 1285,72 & 17,5 & 74,98 & 30,76 & 44,8 & 150,53 \\
\hline 7 espécies & \multicolumn{9}{|c|}{$240,0 \mathrm{~g} \mathrm{ha}^{-1}$ de chlorpyrifos $+48,0 \mathrm{~g} \mathrm{ha}^{-1}$ de nicosulfuron } \\
\hline Boehavia diffusa & 39 & 4 & 1,00 & 1392,86 & 9,75 & 21,05 & 58,21 & 49,58 & 128,84 \\
\hline Commelina benghalensis & 9 & 4 & 1,00 & 321,43 & 2,25 & 21,05 & 13,43 & 11,44 & 45,93 \\
\hline Outras espécies & 19 & 11 & 2,75 & 678,56 & 7,67 & 57,89 & 28,36 & 38,98 & 125,23 \\
\hline 11 espécies & \multicolumn{9}{|c|}{$240,0 \mathrm{~g} \mathrm{ha}^{-1}$ de chlorpyrifos $+64,0 \mathrm{~g} \mathrm{ha}^{-1}$ de nicosulfuron } \\
\hline Boehavia diffusa & 53 & 4 & 1,00 & 1892,86 & 13,25 & 20,00 & 61,63 & 41,73 & 123,36 \\
\hline Commelina benghalensis & 14 & 4 & 1,00 & 500,00 & 3,50 & 20,00 & 16,28 & 11,02 & 47,30 \\
\hline Outras espécies & 19 & 12 & 3 & 678,55 & 15 & 60 & 22,09 & 47,24 & 129,33 \\
\hline
\end{tabular}

(1) Plântulas: total de espécies presentes por tratamento avaliado, evidenciando-se duas com maior índice de valor de importância (IVI). NTI: número total de indivíduos, NPP: número de parcelas presentes, FRE: frequência, DEN: densidade, ABU: abundância, FRR: frequência relativa, DER: densidade relativa, ABR: abundância relativa e IVI: índice de valor de importância; NTI: unidades; NPP: unidades; FRE: ocorrência/total de parcelas; DEN: plântulas/m²; ABU: NTI/NPP; FRR: (FRE x 100) $\Sigma$ FRE; DER: (DEN x 100) $\Sigma$ DEN; ABR: (ABU x 100) $\Sigma$ ABU; IVI: FRR + DER + ABR. 
continham o produto, mesmo na menor dose testada. Entre as observações, destaca-se a densidade (DEN) das espécies vegetais, variável esta que foi mais importante na determinação da importância das espécies. Optou-se pela apresentação das duas espécies de maior importância em cada tratamento, sendo as demais agrupadas como "outras espécies" (Quadro 1). Em solo sem aplicação de inseticida, B. diffusa (ervatostão) foi a espécie mais frequente, apresentando também densidade superior em todas as parcelas com o herbicida, resultando no maior índice de valor de importância (IVI = 136). Neste trabalho, as demais espécies de maior importância (IVI > 30) foram Mollugo verticillata (molugo), Commelina benghalensis (trapoeraba), Euphorbia heterophylla (leiteiro) e Amaranthus hybridus (caruru-roxo) (Quadro 1). Entre essas, C. benghalensis aparece com maior frequência que as demais.

Foi observado decréscimo na taxa de respiração basal do solo com o aumento da dose aplicada do nicosulfuron associado ao chlorpyrifos, sem efeito na ausência do inseticida. Com o aumento de cada unidade (g) na dose do herbicida aplicado ao solo, a respiração diminuiu a uma taxa de $0,0011 \mathrm{mg} \mathrm{dia}^{-1}$, evidenciado pelo coeficiente angular da reta $(\mathrm{p}<0,05)$ (Figura 2$)$. Para o solo isento de inseticida, não foi observada variação nos valores de $\mathrm{CO}_{2}$, permanecendo a taxa fixa de $0,185 \mathrm{mg}$ de $\mathrm{C}$ na forma de $\mathrm{CO}_{2}$ por dia, independentemente da dose aplicada do nicosulfuron (Figura 2).

Avaliando o $\mathrm{C}$ da biomassa microbiana (CBM), verificou-se efeito negativo do herbicida sobre os microrganismos do solo, de maneira mais acentuada quando em mistura com o chlorpyrifos. Em ambos os casos, o decréscimo no CBM foi linear, porém a uma taxa 4,5 vezes maior para as amostras de solo que receberam a aplicação simultânea de inseticida e herbicida (Figura 3). Enquanto para as amostras

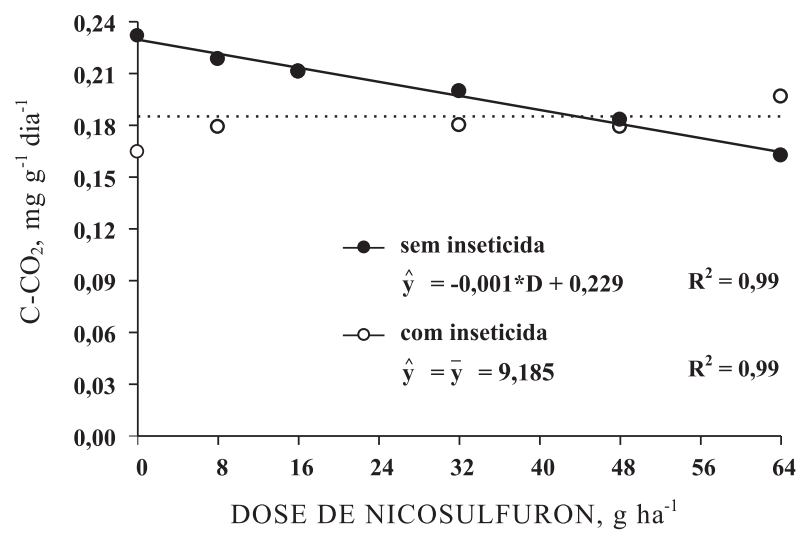

Figura 2. Atividade respiratória basal do solo determinada em amostras de solo, 75 dias após a aplicação de doses do herbicida nicosulfuron, combinadas ou não com o inseticida chlorpyrifos $\left(240 \mathrm{~g} \mathrm{ha}^{-1}\right) . \mathrm{D}=$ doses; *: significativo pelo teste $\mathrm{t}$ a $5 \%$. isentas de agrotóxicos o valor de CBM foi de 321,45 $\mathrm{mg} \mathrm{g}^{-1}$ de $\mathrm{C}$, no solo que recebeu a maior combinação (64 $\mathrm{g} \mathrm{ha}^{-1}$ de nicosulfuron $+240 \mathrm{~g} \mathrm{ha}^{-1}$ de chlorpyrifos) esse valor diminuiu para cerca de $29 \mathrm{mg} \mathrm{g}^{-1}$ de C (Figura 3).

Relacionando-se o C- $\mathrm{CO}_{2}$ com o $\mathrm{CBM}\left(\mathrm{qCO}_{2}\right)$, para amostras de solo tratadas com o nicosulfuron e chlorpyrifos, observou-se aumento linear de 3 × 10-6 unidades por $\mathrm{g} \mathrm{ha}^{-1}$ de nicosulfuron adicionado ao solo (Figura 4). Na ausência do inseticida observou-se aumento nesse indicador, porém a uma taxa 10 vezes menor (Figura 4), indicando maior perda de $\mathrm{C}$ quando o inseticida e herbicida em estudo foram aplicados em sequência.

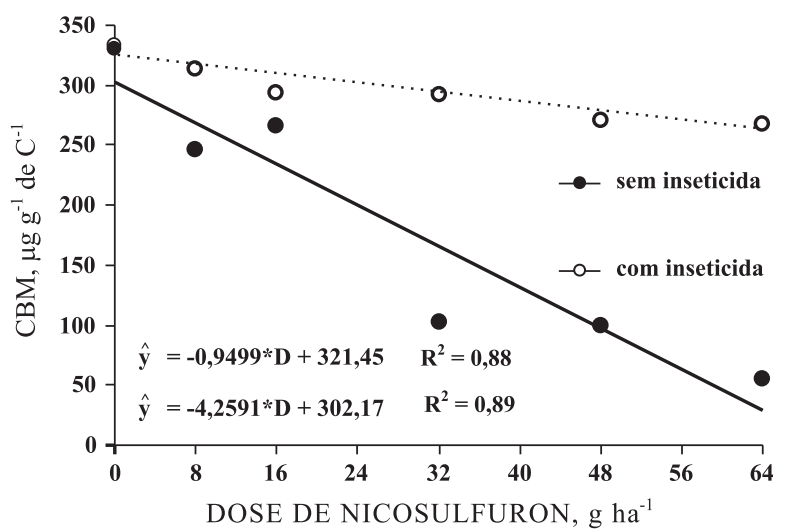

Figura 3. Carbono da biomassa microbiana (CBM) de amostras de solo, 75 dias após a aplicação de doses do herbicida nicosulfuron em diferentes doses, isolado ou simultaneamente ao inseticida chlorpyrifos $\left(240 \mathrm{~g} \mathrm{ha}^{-1}\right)$. D = doses; *: significativo pelo teste t a $5 \%$.

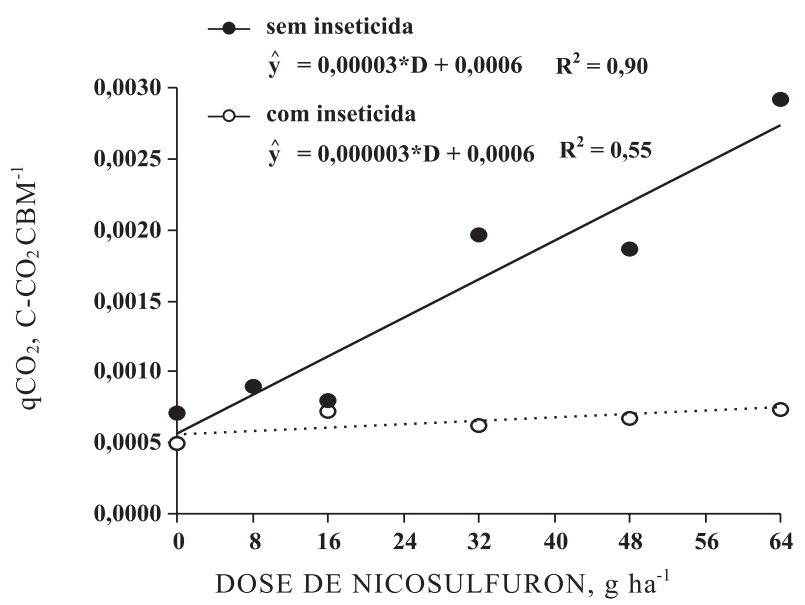

Figura 4. Quociente metabólico de amostras de solo determinado 75 dias após a aplicação de doses do herbicida nicosulfuron em diferentes doses, isolado ou simultaneamente ao inseticida chlorpyrifos $\left(240 \mathrm{~g} \mathrm{ha}^{-1}\right) . \mathrm{D}=$ doses; $* * \mathrm{e} *$ : significativo pelo teste t a 10 e $5 \%$, respectivamente. 


\section{DISCUSSÃO}

A mistura chlorpyrifos e nicosulfuron não provocou mudanças na produção de massa secas total de plântulas avaliadas até a dose aproximada de $20 \mathrm{~g} \mathrm{ha}^{-1}$ do herbicida, a partir da qual a massa foi reduzida (Figura 1). No entanto, esse resultado pode ser pouco importante se for considerado que no sistema de integração lavoura-pecuária o nicosulfuron é recomendado na dose de 4 a $8 \mathrm{~g} \mathrm{ha}^{-1}$ para retardar o crescimento da braquiária (Jakelaitis et al., 2005; Alvarenga et al., 2006). Todavia, ao considerar a recomendação de uso desse produto de $40 \mathrm{~g} \mathrm{ha}^{-1}$ para o controle de plantas daninhas (Rodrigues \& Almeida, 2005), a aplicação simultânea do chlorpyrifos altera negativamente a reinfestação da área a partir de plântulas do banco de sementes do solo.

O elevado índice de valor de importância (IVI) da espécie $B$. diffusa pode ser devido ao aumento da concentração do nicosulfuron no solo (Quadro 1), impedindo a germinação de outras espécies e diminuindo a competição interespecífica. Nessa circunstância, a diminuição do número de espécies na área faz com que aquelas predominantes tenham maior capacidade de crescimento e desenvolvimento e, consequentemente, maior produção de biomassa. Por outro lado, não se descarta a hipótese de que o herbicida tenha efeito negativo sobre algum fator estressante à germinação e emergência de $B$. diffusa, funcionando como um estimulante indireto na germinação.

Com a aplicação do chlorpyrifos, o IVI de $B$. diffusa foi baixo (IVI = 41) somente na dose zero de nicosulfuron (Quadro 1). Fica claro o efeito tóxico residual do herbicida quando são comparadas as parcelas que receberam o produto com aquelas isentas deste. O número total de indivíduos (NTI) nos dois tratamentos sem herbicida foi de 397 e 518 plântulas por vaso, respectivamente, para amostras com e sem aplicação do inseticida. Nas parcelas com nicosulfuron, o NTI não ultrapassou 150 plântulas (Quadro 1). De maneira geral, o grupo químico ao qual este produto pertence (sulfonilureias) apresenta persistência moderada a longa no solo, agindo em baixas doses, fisiologicamente, por meio da inibição da síntese dos aminoácidos valina, leucina e isoleucina (Bridges, 2003; Thill, 2003).

Em outros trabalhos já foram observados efeitos tóxicos em plantas sob efeito da aplicação de inseticidas fosforados após os herbicidas inibidores da ALS (Diehl et al., 1995; Trezzi et al., 2005). As causas incluem a possível redução na deposição de cera epicuticular em folhas de plantas sob efeito do inseticida fosforado (Diehl et al., 1995) ou redução da atividade da citocromo $\mathrm{P} 450$, enzima responsável pela metabolização do nicosulfuron (Brown, 1990).

A aplicação de agrotóxicos no solo influencia toda a dinâmica do ecossistema, agindo sobre as formas de vida e suas relações. O decréscimo na taxa de respiração basal do solo a partir da aplicação das doses do nicosulfuron, associadas ao chlorpyrifos (Figura 2), pode ser interpretado como impacto indicativo de elevada atividade biológica, característica desejável por significar degradação de resíduos orgânicos (incluindo agrotóxicos) a nutrientes disponíveis para as plantas; ou, ainda, como impacto negativo, resultado de estresse metabólico na microbiota, devido à presença de moléculas tóxicas, sendo desfavorável para muitos processos físico-químicos, como agregação, capacidade de troca de cátions e retenção de água (Tótola \& Chaer, 2002). Assim, o maior desprendimento de $\mathrm{C}-\mathrm{CO}_{2}$ pode indicar tanto um distúrbio ecológico como um alto nível de produtividade do ecossistema (Tótola \& Chaer, 2002). Nesta pesquisa, a aplicação somente do herbicida não provocou mudanças na taxa de respiração basal, independentemente da dose utilizada. Esse resultado não significa ausência de efeitos sobre os microrganismos do solo, e sim constatação de que esta variável - taxa de respiração basal do solo - não constitui indicador sensível à presença do herbicida, devendo, portanto, ser avaliada em conjunto com outros indicadores.

Em outros trabalhos também foi observado que a aplicação de herbicidas ao solo, apesar de não alterar a taxa de respiração basal, provocou mudanças na quantidade de microrganismos (Santos et al., 2007). Isso pode ser devido ao estresse promovido pelo xenobiótico à biomassa de microrganismos, fazendo com que aumentem a respiração enquanto perdem massa na forma de $\mathrm{CO}_{2}$ (Tótola \& Chaer, 2002). Dessa forma, com a diminuição da massa de microrganismos e o aumento da respiração, tem-se menor eficiência no uso do $\mathrm{C}$ sem alterações no desprendimento de $\mathrm{CO}_{2}$.

Para contornar possíveis interpretações errôneas a partir dos resultados da respiração basal, faz-se necessária sua avaliação juntamente com outros indicadores, principalmente a biomassa de microrganismos $-\mathrm{C}$ da biomassa microbiana (CBM). Assim, observou-se decréscimo no CBM em todos os tratamentos com os produtos, porém de maneira mais acentuada para as amostras de solo tratadas com a mistura de inseticida ao herbicida (Figura 3).

Esse efeito negativo do herbicida sobre a biomassa dos microrganismos pode ser o resultado direto da intoxicação do princípio ativo testado (nicosulfuron) sobre seu metabolismo e, ou, efeito dos constituintes do produto comercial. Nesse caso, a mistura dos produtos provoca efeito sinergístico, pois a soma dos efeitos de diminuição na biomassa entre doses do herbicida e do inseticida é menor do que o valor observado no primeiro caso (Figura 3). Segundo Malkones (2000), há incoerência nos ensaios biológicos para testes toxicológicos de moléculas de agrotóxicos a organismos - não alvo, uma vez que são realizados com o produto técnico e, após registro deste no órgão competente, o que se lança no ambiente são produtos formulados. Em trabalho realizado por Santos et al. (2005), testando a sensibilidade de três estirpes de Bradyrhizobium a oito formulações comerciais de 
glyphosate, foi observado que o ingrediente ativo, isento de qualquer adjuvante, causou menor intoxicação, comparado a qualquer produto formulado. Outra possibilidade - já apresentada para explicar os resultados observados em plantas - é a alteração enzimática provocada pelo inseticida ao complexo citocromo P450, como ocorre na constituição da família de heme proteínas presentes também em bactérias e fungos (Berger, 2002).

Para melhor entender o efeito dos produtos sobre a atividade microbiana, propõe-se a avaliação conjunta do desprendimento de $\mathrm{CO}_{2}$ e do CBM, em forma de relação, por meio do quociente metabólico $\left(\mathrm{qCO}_{2}\right)$ (Anderson \& Domsch, 1985). A avaliação do $\mathrm{qCO}_{2}$ confirmou o efeito negativo da aplicação sequencial do inseticida e do herbicida. A relação entre $\mathrm{C}-\mathrm{CO}_{2}$ e $\mathrm{CBM}$ aumentou a uma taxa linear de $3 \times 10^{-5}$ unidades por $\mathrm{g} \mathrm{ha}^{-1}$ de nicosulfuron adicionado ao solo (Figura 4). Na ausência do inseticida observou-se aumento nesse indicador, porém com acréscimo bem inferior, de $3 \times 10^{-6}$ unidades por $\mathrm{g} \mathrm{ha}^{-1}$ (Figura 4). Comparando-se os coeficientes (angular da reta), em ambas equações ajustadas para os valores observados para qCO2, observa-se taxa superior para os tratamentos com aplicação seqüencial de produtos. Dessa forma, percebe-se uma ineficiência no uso do C pelos microrganismos, porém, com taxa 10 vezes superior quando se faz a aplicação seqüencial dos produtos (Figura 4).

Como sugestão de manejo, a substituição do nicosulfuron em lavouras onde se pretende utilizar o inseticida chlorpyrifos para controle de insetos, em função do resíduo do herbicida no solo, pode ser uma prática eficiente, desde que as alternativas de uso não promovam efeito residual ao solo. Outra sugestão é o estudo de novos inseticidas (triflumuron e tebufenozid, entre outros) para as aplicações sequenciais a herbicidas pertencentes ao grupo químico das sulfonilureias.

\section{CONCLUSÕES}

1. A aplicação de nicosulfuron e chlorpyrifos testados ao solo promove alteração na composição fitossociológica das plântulas emergidas.

2. O nicosulfuron, independentemente da dose testada, não altera a respiração basal do solo, porém diminui o $\mathrm{C}$ da biomassa microbiana.

3. A aplicação sequencial do nicosulfuron e chlorpyrifos apresenta efeito sinergístico, alterando negativamente a respiração basal e o $\mathrm{C}$ da biomassa microbiana do solo.

4. A ineficiência dos microrganismos em utilizar o C do meio é maior quando ao solo se faz a aplicação sequencial dos produtos testados, comparado à soma dos efeitos da aplicação isolada.

\section{AGRADECIMENTOS}

À Fundação de Amparo à Pesquisa do Estado de Minas Gerais (FAPEMIG) e ao Conselho Nacional de Desenvolvimento Científico e Tecnológico (CNPq), pelo apoio financeiro à realização desta pesquisa.

\section{LITERATURA CITADA}

ALVARENGA, C.R.; COBUCCI, T.; KLUTHCOUSKI, J.; WRUCK, F.J.; CRUZ, J.C. \& GONTIJO NETO, M.M. A cultura do milho na integração lavoura-pecuária. Sete Lagoas, Embrapa, 2006. (Circular Técnica)

ANDERSON, J.P.E. Soil respiration. In: PAGE, A.L.; MILLER, R.H. \& KEENEY, D.R., eds. Methods of soil analysis; Chemical and microbiological properties. Madison, America Society of Agronomy, 1982. Part 2. p.831-871.

ANDERSON, T.H. \& DOMSCH, K.H. Determination of ecophysiological maintenance carbon requirements of soil microorganisms in a dormant state. Biol. Fert. Soils, 1:8189, 1985.

BERGER, R.G.; KRINGS, U. \& ZORN, H. Biotechnological flavour generation. In: TAYLOR, A., ed. Food flavour technology. Sheffield, Sheffield Academic Press, 2002. p.60-104.

BRIDGES, D.C. Glyphosate-type herbicides. In: BRIDGES, D.C. Herbicide action course. West Lafayette, Purdue University, 2003. p.501-513.

BROWN, H.M. Mode of action, crop selectivity, and soil relations of the sulfonylurea herbicides. Pest. Sci., 29:263-281, 1990.

CHAER, G.M. \& TÓTOLA, M.R. Impacto do manejo de resíduos orgânicos durante a reforma de plantios de eucalipto sobre indicadores de qualidade do solo. R. Bras. Ci. Solo, 31:13811396, 2007.

DIEHL, K.E.; TAYLOR, S.L.; SIMPSON, D.M. \& STOLLER, E.W. Effect of soil organic matter on the interaction between nicosulfuron and terbufos in corn (Zea mays). Weed Sci., 43:306-311, 1995.

FERREIRA, A.S.; CAMARGO, F.A.O. \& VIDOR, C. Utilização de micro-ondas na avaliação da biomassa microbiana no solo. R. Bras. Ci. Solo, 23:991-996, 1999.

JAKELAITIS, A.; SILVA, A.A.; FERREIRA, L.R.; SILVA, A.F.; PEREIRA, J.L. \& VIANA, R.G. Efeitos de herbicidas no consórcio de milho com Brachiaria brizantha. Planta Daninha, 23:69-78, 2005.

JAKELAITIS, A.; SANTOS, J.B.; VIVIAN, R. \& SILVA, A.A. Atividade microbiana e produção de milho (Zea mays) e de Brachiaria brizantha sob diferentes métodos de controle de plantas daninhas. Planta Daninha, 25:71-78, 2007.

ISLAM, K.R. \& WEIL, R.R. Microwave irradiation of soil for routine measurement of microbial biomass carbon. Biol. Fert. Soils, 27:408-416, 1998. 
MALKONES, H.P. Comparasion of the effects of differently formulated herbicides on soil microbial activities - A review. J. Plant Disease Protec., 8:781-789, 2000.

MONQUERO, P.A. \& CHRISTOFFOLETI, P.J. Banco de sementes de plantas daninhas e herbicidas como fator de seleção. Bragantia, 64:203-209, 2005.

MOREIRA, F.L.; OLIVEIRA, S.J.; ARAÚJO, J.G.F. \& BRAGA, M.G. Impacto ambiental e administração de problemas toxicológicos na utilização de inseticidas agrícolas. Cader. Adm. Rural, 8:28-31, 1996.

MUELLER-DOMBOIS, D. \& ELLENBERG, H. A. Aims and methods of vegetation ecology. New York, John Wiley, 1974. $547 \mathrm{p}$

NICOLAI, M.; CARVALHO, S.J.P.; LÓPEZ-OVEJERO, R.F. \& CHRISTOFFOLETI, P.J. Aplicação conjunta de herbicidas e inseticidas na cultura do milho. Bragantia, 65:413-420, 2006.

OLIVEIRA, C.N.; NEVES, P.M.O.J. \& KAUASOE, L.S. Compatibility between the entomopathogenic fungus Beauveria bassiana and insecticides used in coffee plantations. Sci. Agríc., 60:663-667, 2003.

PEREIRA, J.L.; SILVA, A.A.; PICANÇO, M.C.; BARROS, E.C. \& JAKELAITIS, A. Effects of herbicide and insecticide interaction on soil entomofaune under maize. Crop. J. Environ. Sci. Health, B40:43-52, 2005.

PEREIRA, A.A.; HUNGRIA, M.; FRANCHINI, J.C.; KASCHUK, G.; CHUEIRE, L.M.O.; CAMPO, R.J. \& TORRES, E. Variações qualitativas e quantitativas na microbiota do solo e na fixação biológica do nitrogênio sob diferentes manejos com soja. R. Bras. Ci. Solo, 31:1397$1412,2007$.

RANGEL, O.J.P. \& SILVA, C.A. Estoques de carbono e nitrogênio e frações orgânicas de Latossolo submetido a diferentes sistemas de uso e manejo. R. Bras. Ci. Solo, 31:1609-1631, 2007.
RODRIGUES, B.N. \& ALMEIDA, F.S. Guia de herbicidas. 5.ed. Londrina, Grarfmake, 2005. 591p.

SANTOS, J.B.; JAKELAITIS, A.; SILVA, A.A.; VIVIAN, R.; COSTA, M.D. \& SILVA, A.F. Atividade microbiana do solo após aplicação de herbicidas em sistemas de plantio direto e convencional. Planta Daninha, 23:683-691, 2005.

SANTOS, J.B.; FERREIRA, E.A.; REIS, M.R.; SILVA, A.A.; FIALHO, C.M.T. \& FREITAS, M.A.M. Avaliação de formulações de glyphosate sobre soja roundup ready. Planta Daninha, 25:165-171, 2007.

SILVA, A.A.; FREITAS, F.M.; FERREIRA, L.R. \& JAKELAITIS, A. Efeitos de mistura de herbicida com inseticida sobre cultura do milho, as plantas daninhas e a lagarta-docartucho. Planta Daninha, 23:517-525, 2005.

TEMPLETON, A.R. \& LEVIN, D.A. Evolutionary consequences of seed pools. Am. Naturalist, 114:232-249, 1979.

THILL. D.C. Lipid biosyntesis inhibitors. In: BRIDGES, D.C. Herbicide action course. West Lafayette, Purdue University, 2003. p.293-347.

TÓTOLA, M.R. \& CHAER, G.M. Microrganismos e processos microbiológicos como indicadores da qualidade dos solos. In: ALVAREZ V.,V.H.; SCHAEFER, C.E.G.R.; BARROS, N.F.; MELLO, J.W.V. \& COSTA, L.M., eds. Tópicos em ciência do solo.Viçosa, MG, Sociedade Brasileira de Ciência do Solo, 2002. v.2. p.195-276.

TREZZI, M.M.; FELIPPI, C.L.; NUNES, A.L.; CARNIELETO, C.E. \& FERREIRA, A.R.J. Weed control efficacy and corn toxicity of the foramsulfuron and iodosulfuron mixture, alone or combined with atrazine and/or chlorpyriphos. Planta Daninha, 23:653-659, 2005.

VANCE, E.D.; BROOKES, P.C. \& JENKINSON, D.S. An extraction method for measuring soil microbial biomass C. Soil Biol. Biochem., 19:703-707, 1987. 